\title{
Reliability Analysis of a Discrete Life Time Model
}

\author{
Sandeep kumar \\ Hindu College \\ University of Delhi
}

\author{
Birjesh Kumar, Ph.D \\ J.P Institute of Engg.\& \\ Technology, Meerut
}

\author{
Alka Chaudhary, Ph.D \\ Meerut College, Meerut \\ C.C.S. University, Meerut
}

\begin{abstract}
Several studies deals with life testing of various systems with respect to Reliability characteristics. The life testing generally considered a continuous life time Distribution. However, there are situations when life times are recorded on discrete scale. In life testing, Geometric distribution has an important role in such type of analysis. A vast literature on the life testing plans in the Bayesian framework is also available where the parameter of basic life time distribution is considered as a random variable. The present study deals with the development of the methodology for life testing in terms of classical, modified classical and Bayes Reliability.
\end{abstract}

\section{Keywords}

Classical Component Reliability ( CCR),Modified Classical Component Reliability(CCR*), Bayes Component Reliability(B C R).

\section{INTRODUCTION}

Reliability theory a branch of statistical science, has an effective role in the rapid advancement in human amenities, such as modern electronic computers, televisions, washing machines, electronic locomotives, refrigerators, mobile and cell phones, etc. When a manufacturer floats a new brand of light bulb in the market, he would like his customers to have some information about the average life of his product. Life testing experiments are designed to measure the average life of the component or to answer such questions as "what is the probability that the item will fail in the time interval [ $t_{0}$, $t_{0}+t$ ] given that it was working at time $t_{0}$ ". In simple life testing experiments a number of items are subject to tests and the data consist of the recorded lives of all or some of the items. Studies in life testing generally consider a continuous lifetime distribution.

However, there are situations when lifetimes are recorded on a discrete scale. For example, the lifetime of light bulb lights up whenever a paper enters the machine i.e. the bulb functions at discrete time epochs and can fail after providing a certain number of prints. In this case, the lifetime is defined as the number of successful operation of a device before failure. Obviously, the geometric distribution has an important place in such analysis.

Life testing is a costly and time consuming phenomenon, and, therefore, it should be recognized that the parameters, characterizing reliability characteristics, in a life time distribution are bound to follow some random variations due to environmental changes. So it is a factor which should be considered with experimental data for analyzing the reliability characteristics of the systems. Obviously, the Bayesian analysis of the various reliability characteristics of systems becomes important. A comparative study in this regard is by Martz and Walher [1982]. Bhattacharya [1967] presented the Bayesian analysis of the system reliability using many prior distributions. Studies like Brush [1986], Brush et, al [1981], and Sharma et., Al.[1992, 1993, 1994, 2005]are also efforts in the same direction following the concepts, posterior analysis of some other reliability characteristics like availability, hazard functions, MTSF etc. of certain complex static system will be considered in the study.

\section{NOTATIONS}

MTSF : Mean time to system failure

$R(t)=P(X>t):$ Reliability of Independent and identical components for a mission time $\mathrm{t}$.

CCR : Classical Component Reliability.

CCR* : Modified Classical Component Reliability.

BCR : Bayes Component Reliability .

$R_{k m}(t)$ : Classical reliability of a k-out of m system.

$\mathrm{R}^{*}{ }_{k m}(\mathrm{t})$ : Modified Classical Reliability of a k-out of $\mathrm{m}$.

$\mathrm{BR}_{k m}(\mathrm{t})$ : Bayes Reliability of a k-out of m system.

$\mathrm{R}_{\mathrm{S}}(\mathrm{t})$ : Classical Reliability of a series system.

$\mathrm{R}_{\mathrm{S}}(\mathrm{t})$ : Modified Classical Reliability of a series system.

$\mathrm{BR}_{\mathrm{S}}(\mathrm{t})$ : Bayes Reliability of a series system.

$\mathrm{R}_{\mathrm{P}}(\mathrm{t})$ : Classical Reliability of a parallel system.

$\mathrm{R}{ }_{\mathrm{P}}(\mathrm{t})$ :Modified Classical Reliability of a parallel system.

$\mathrm{B}_{\mathrm{P}}(\mathrm{t})$ : Bayes Reliability of a parallel system.

\section{STATISTICAL BACKGROUND}

For developing the procedure, it is assume that-

(a) Suppose a lot of $\mathrm{N}$ items, is to be tested with a life testing operation. Let the life time distribution of each items be geometric with p.m.f.

$$
f(X, \theta)=(1-\theta) \cdot \theta^{x} ; \mathrm{x}=0,1,2 \ldots
$$

$0<\theta<1$

With

$$
\begin{aligned}
& \mathrm{MTSF}=\mathrm{E}[\mathrm{X}]=\frac{\theta}{(1-\theta)} ; \\
& \mathrm{V}[\mathrm{X}]=\frac{\theta}{(1-\theta)^{2}}
\end{aligned}
$$




$$
\begin{aligned}
& \mathrm{R}(\mathrm{t})=P[X \geq t]=\theta^{t}, \quad ; t=0,1,2 \ldots \ldots . . . \\
& \mathrm{h}(\mathrm{t})=(1-\theta) \\
& \mathrm{CV}=\frac{1}{\sqrt{\theta}} \times 100
\end{aligned}
$$

Let $\theta$ be reliability standard and the point $\theta=\theta_{0}$ on this scale represent the MTSF level assigned by investigator, called the acceptance mean level.

(b) The parameter $\theta$ is considered as a random variable with and its prior is beta distribution of fist kind with p.d.f. is -

$$
g(\theta)=\frac{\theta^{U-1}(1-\theta)^{V-1}}{B(U, V)} ; U, V>0 ; 0<\theta<1
$$

\section{CLASSICAL COMPONENT}

\section{RELIABILITY (CCR)}

The classical component reliability (CCR) of a discrete life time model is given by

$$
C C R=P\left[X \geq t \mid \theta=\theta_{0}\right]
$$

Classical component reliability is defined as the probability that the system will survive up to time $t$, when its reliability standard is $\theta_{0}$

With

$$
\begin{aligned}
& \mathrm{E}[\mathrm{X}]=\frac{U}{U+V}, \\
& \mathrm{~V}[\mathrm{X}]=\frac{U}{(\mathrm{U}+V)^{2}(U+V+1)}
\end{aligned}
$$

\section{MODIFIED CLASSICAL COMPONENT RELIABILITY (CCR*)}

It is defined as-

$$
\begin{aligned}
& C C R^{*}=P\left[X \geq t \mid \theta \leq \theta_{0}\right] \\
& =\frac{P\left[X \geq t, \theta \leq \theta_{0}\right]}{P\left(\theta \leq \theta_{0}\right)}=\frac{\int_{0}^{\theta_{0}} R(\theta) \cdot g(\theta) \cdot d \theta}{\int_{0}^{\theta_{0}} g(\theta) \cdot d \theta}
\end{aligned}
$$

\section{BAYES COMPONENT RELIABILITY (BCR)}

In difference to conditioning on an unknown even $\left(\theta=\theta_{0}\right)$, as is done in the classical measure of reliability, the Bayes component reliability conditions as given in Brush (1986) on the actual data observed. The definition of the Bayes component reliability (BCR) is

$$
P\left[\theta \leq \theta_{0} \mid X \geq t\right]=B C R
$$

$$
\Rightarrow B C R=\frac{P\left[X \geq t, \theta \leq \theta_{0}\right]}{P[X \geq t, \theta \geq 0]}=\frac{\int_{0}^{\theta_{0}} R(\theta) \cdot g(\theta) \cdot d \theta}{\int_{0}^{1} R(\theta) \cdot g(\theta) \cdot d \theta}
$$

Where $R(\theta)=P[X \geq t \mid \theta]$ and $g(\theta)$ is the prior p.d.f. of $\theta$. Thus on using various definitions and mathematical steps, we are able to say that BCR is nothing but the conditional probability of the system that the reliability being better than a standard level (AML) given that system will survive at least time $\mathrm{t}$.

\section{COMPARISON BETWEEN CLASSICAL, MODIFIED CLASSICAL \& BAYES RELIABILITY UNDER PRIOR INFORMATION}

For the purpose of making comparisons between the classical and Bayes definitions of system reliability, an additional concept is introduced.

So how do $\mathrm{CCR}^{*}$ (4) and BCR (6) compare? Now using equations (4) and (6)

$$
\frac{B C R}{C C R}=\frac{\mathrm{P}\left(\theta \leq \theta_{0}\right)}{\mathrm{P}(X \geq t)}
$$

We conclude that if the reliability of the system is better than standard level $\left(\theta_{0}\right)$ than $\mathrm{P}\left(\theta \leq \theta_{0}\right)$ is high and $\mathrm{P}(X \geq t)$ is low, so BCR will be greater than CCR. On the other hand, if the reliability level of the system is worse than standard then $P\left(\theta \leq \theta_{0}\right)$ is low and $P(X \geq t)$ is high. In such case BCR is less than CCR* The changing relationship between BCR and CCR ${ }^{*}$, shown in table 1 - table 3, It also calculates the various reliability characteristics. Each of two definitions of reliability is useful for analyzing the discrete life time model. The classical and modified classical reliability help to answer the important questions such as following.

1. If the system runs exactly at the reliability standard all of the time, what percentage of the system will be failed?

2. If the system mean reliability varies randomly, what percentage of the system will be failed for these instance when reliability is better than its standard, which simply mean BCR > CCR*

The Bayes measure of reliability (BCR) gives answer to the important questions, such that if the system fails, what is the probability that the reliability is of substandard form. 


\section{CLASSICAL, MODIFIED CLASSICAL AND BAYES RELIABILITY OF K-OUT OF M, SERIES AND PARALLEL SYSTEM}

(a) With simple probabilistic reasoning, the reliability of a k-out of m system, say $R_{k m}(t)$, is given by

$$
R_{k m}(t)=\sum_{i=k}^{m}\left(\begin{array}{c}
m \\
i
\end{array}\right) \cdot\left[R_{c}(t)\right]^{i}\left[1-R_{c}(t)\right]^{m-i}
$$

Where, $R_{c}(t)$ stands for the component reliability,

Obviously

Classical reliability of a k-out of m-system is given by

$$
C R_{k m}(t)=\sum_{i=k}^{m}\left(\begin{array}{l}
m \\
i
\end{array}\right)[C C R]^{i}[1-C C R]^{m-i}
$$

Modified classical reliability of the system will be

$$
C R_{k m}^{*}(t)=\sum_{i=k}^{m}\left(\begin{array}{l}
m \\
i
\end{array}\right)\left[C C R^{*}\right]^{i}\left[1-C C R^{*}\right]^{m-i}
$$

Bayes reliability of the k-out of $\mathrm{m}$ system is

$$
B R_{k m}(t)=\sum_{i=k}^{m}\left(\begin{array}{l}
m \\
i
\end{array}\right)[B C R]^{i}[1-B C R]^{m-i}
$$

(b) Putting $\mathrm{k}=\mathrm{m}$ is (8), one gets the reliability of a series system, say $R_{s}(t)$ as

$$
R_{s}(t)=\left[R_{c}(t)\right]^{m}
$$

where, $R_{c}(t)$ is the component reliability. Thus we get classical reliability of a series system $R_{s}(t)$ is

$$
C R_{s}(t)=[C C R]^{m}
$$

Modified classical reliability of the series system is

$$
C R_{s}^{*}(t)=\left[C C R^{*}\right]^{m}
$$

and

Bayes reliability of the system is given by

$$
B R_{s}(t)=[B C R]^{m}
$$

(c) Similarly on putting $\mathrm{k}=1$, in (8), one gets the reliability of a parallel system, say $R_{p}(t)$ is

$$
R_{p}(t)=1-\left[1-R_{c}(t)\right]^{m}
$$

By the same way as defined above, on putting CCR, CCR*, BCR in (15), we gets the classical, modified classical and Bayes reliability of the parallel system respectively.

\section{AN EXAMPLE}

For demonstrating variations in classical, Bayes and Modified classical component reliability as the mean of quality distribution and the mission time varies, we use the expressions in equation (3, 4 and 6). The respective values of CCR, CCR and BCR are given in Table- 1 and Table- 3 , with fix value $\mathrm{U}=1$ and varying $\mathrm{V}=1,2,3,4 \& 5$ and time $(\mathrm{t})=1,2$ and 3 respectively.

Also the performance of parallel, series and $\mathrm{k}$-out of $-\mathrm{m}$ system reliability with respect to CCR, $\mathrm{CCR}^{*}$ and $\mathrm{BCR}$ shows in Table 4-Table 6 , with fix value $\mathrm{U}=1$ and varying $\mathrm{V}=1,2,3$, $4 \& 5$ and time $(t)=1,2$ and 3 respectively

TABLE- $1, t=1$

\begin{tabular}{|c|c|c|c|c|c|}
\hline $\mathbf{U}$ & $\mathbf{V}$ & $\frac{U}{U+V}=E(\theta)$ & $\mathbf{C C R}$ & $\mathbf{C C R}^{*}$ & BCR \\
\hline 1 & 1 & 0.50 & 0.800 & 0.2133 & 0.512 \\
\hline 1 & 2 & 0.33 & 0.800 & 0.1423 & 0.8199 \\
\hline 1 & 3 & 0.25 & 0.800 & 0.0950 & 0.9424 \\
\hline 1 & 4 & 0.20 & 0.800 & 0.0657 & 0.9819 \\
\hline 1 & 5 & 0.17 & 0.800 & 0.0460 & 0.9896 \\
\hline
\end{tabular}

TABLE- $2 \quad t=2$

\begin{tabular}{|c|c|c|c|c|c|}
\hline $\mathbf{U}$ & $\mathbf{V}$ & $E(\theta)=\frac{U}{U+V}$ & $\mathbf{C C R}$ & $\mathbf{C C R}^{*}$ & $\mathbf{B C R}$ \\
\hline 1 & 1 & 0.50 & 0.640 & 0.1280 & 0.4096 \\
\hline 1 & 2 & 0.33 & 0.640 & 0.0769 & 0.7380 \\
\hline 1 & 3 & 0.25 & 0.640 & 0.0454 & 0.8982 \\
\hline 1 & 4 & 0.20 & 0.640 & 0.0276 & 0.9718 \\
\hline 1 & 5 & 0.17 & 0.640 & 0.0175 & 0.9722 \\
\hline
\end{tabular}

TABLE- $3, t=3$

\begin{tabular}{|c|c|c|c|c|c|}
\hline $\mathbf{U}$ & $\mathbf{V}$ & $E(\theta)=\frac{U}{U+V}$ & $\mathbf{C C R}$ & $\mathbf{C C R}^{*}$ & BCR \\
\hline 1 & 1 & 0.50 & 0.512 & 0.0819 & 0.3277 \\
\hline 1 & 2 & 0.33 & 0.512 & 0.0454 & 0.6547 \\
\hline 1 & 3 & 0.25 & 0.512 & 0.0245 & 0.8438 \\
\hline 1 & 4 & 0.20 & 0.512 & 0.0136 & 0.9441 \\
\hline 1 & 5 & 0.17 & 0.512 & 0.0075 & 0.9682 \\
\hline
\end{tabular}


Comparison among Parallel, Series and K out of M System with Reliability

TABLE- $4, t=1$

\begin{tabular}{|c|c|c|c|c|c|c|c|c|c|c|c|}
\hline \multirow[t]{2}{*}{$\mathbf{U}$} & \multirow[t]{2}{*}{$\mathbf{V}$} & \multirow{2}{*}{$E(\theta)=\frac{U}{U+V}$} & \multicolumn{3}{|c|}{ k-out of m system } & \multicolumn{3}{|c|}{ Series system } & \multicolumn{3}{|c|}{ Parallel system } \\
\hline & & & $C R_{k m}^{(t)}$ & $C R_{k m}^{*}$ & $B R_{k m}$ & $C R_{s}(t)$ & $C R_{s}^{*}(t)$ & $B R_{s}(t)$ & $C R_{p}(t)$ & $C R_{p}^{*}(t)$ & $B R_{p}(t)$ \\
\hline 1 & 1 & 0.50 & 0.70951 & 0.11708 & 0.91799 & 0.262 & 0.0097 & 0.13422 & 0.9533 & 0.5131 & 0.88378 \\
\hline 1 & 2 & 0.33 & 0.70951 & 0.0549 & 0.91437 & 0.262 & 0.00291 & 0.55116 & 0.992 & 0.36903 & 0.99415 \\
\hline 1 & 3 & 0.25 & 0.70951 & 0.0254 & 0.99059 & 0.262 & 0.00086 & 0.83829 & 0.992 & 0.25878 & 0.99981 \\
\hline 1 & 4 & 0.20 & 0.70951 & 0.01238 & 0.99903 & 0.262 & 0.00028 & 0.94667 & 0.992 & 0.18443 & 0.99999 \\
\hline 1 & 5 & 0.17 & 0.70951 & 0.00615 & 0.99967 & 0.262 & 0.00009 & 0.96912 & 0.992 & 0.13175 & 0.99999 \\
\hline
\end{tabular}

TABLE-5 , $\mathrm{t}=2$

\begin{tabular}{|c|c|c|c|c|c|c|c|c|c|c|c|}
\hline \multirow[t]{2}{*}{$\mathrm{U}$} & \multirow[t]{2}{*}{ V } & \multirow{2}{*}{$E(\theta)=\frac{U}{U+1}$} & \multicolumn{3}{|c|}{ k-out of m system } & \multicolumn{3}{|c|}{ Series system } & \multicolumn{3}{|c|}{ Parallel system } \\
\hline & & & $C R_{k m}^{(t)}$ & $C R_{k m}^{*}$ & $B R_{k m}$ & $C R_{s}(t)$ & $C R_{s}^{*}(t)$ & $B R_{s}(t)$ & $C R_{p}(t)$ & $C R_{p}^{*}(t)$ & $B R_{p}(t)$ \\
\hline 1 & 1 & 0.50 & 0.5169 & 0.044957 & 0.36587 & 1.342 & 0.00209 & 0.06872 & 0.8831 & 0.33694 & 0.7942 \\
\hline 1 & 2 & 0.33 & 0.5169 & 0.01683 & 0.83003 & 1.342 & 0.00045 & 0.40194 & 0.8831 & 0.21341 & 0.9820 \\
\hline 1 & 3 & 0.25 & 0.5169 & 0.00599 & 0.97102 & 1.342 & 0.00009 & 0.72463 & 0.8831 & 0.13011 & 0.9984 \\
\hline 1 & 4 & 0.20 & 0.5169 & 0.00224 & 0.99765 & 1.342 & 0.00002 & 0.91776 & 0.8831 & 0.08054 & 0.9999 \\
\hline 1 & 5 & 0.17 & 0.5169 & 0.00091 & 0.99772 & 1.342 & 0.00001 & 0.91889 & 0.8831 & 0.05158 & 0.9999 \\
\hline
\end{tabular}

\section{TABLE-6, $\mathrm{t}=3$}

\begin{tabular}{|c|c|c|c|c|c|c|c|c|c|c|c|}
\hline U & \multirow{2}{*}{} & & \multirow{2}{*}{$E(\theta)=\frac{U}{U+V}$} & \multicolumn{3}{|c|}{ k-out of m system } & \multicolumn{3}{c|}{ Series system } & \multicolumn{3}{c|}{ Parallel system } \\
\cline { 4 - 12 } & & & $C R_{k m}^{(t)}$ & $C R_{k m}^{*}$ & $B R_{k m}$ & $C R_{s}(t)$ & $C R_{s}^{*}(t)$ & $B R_{s}(t)$ & $C R_{p}(t)$ & $C R_{p}^{*}(t)$ & $B R_{p}(t)$ \\
\hline 1 & 1 & 0.50 & 0.5179 & 0.01902 & 0.25178 & 0.1342 & 0.00054 & 0.0352 & 0.8837 & 0.22612 & 0.69612 \\
\hline 1 & 2 & 0.33 & 0.5179 & 0.00599 & 0.72464 & 0.1342 & 0.00009 & 0.2806 & 0.8837 & 0.13011 & 0.95883 \\
\hline 1 & 3 & 0.25 & 0.5179 & 0.00177 & 0.93442 & 0.1342 & 0.00003 & 0.6008 & 0.8837 & 0.07171 & 0.99618 \\
\hline 1 & 4 & 0.20 & 0.5179 & 0.00055 & 0.99106 & 0.1342 & 0.000002 & 0.8423 & 0.8837 & 0.04024 & 0.99981 \\
\hline 1 & 5 & 0.17 & 0.5179 & 0.00016 & 0.99703 & 0.1342 & 0.000001 & 0.9076 & 0.8837 & 0.02233 & 0.99996 \\
\hline
\end{tabular}




\section{CONCLUSION}

After highlighting some of the concepts regarding system quality by using reliability function, where time is considered as a variable in the Bayesian setup, a methodology is developed to deal with the phenomenon by using classical, modified classical and Bayesian methods with reliability estimation based on Bayesian information. Subsequently, it is shown as to how we can use the Bayesian information to system quality improvement, various tables can be analyzed to see the comparison between classical, modified classical and Bayesian reliability.From table 1, 2, 3, 4, 5 and 6, It is seen that (i) As usual the reliability for different static system models decreases uniformly as the mission time (t) increases.(ii) Bayesian reliability is consistent as compared to classical and modified classical reliability with increasing time (t) The conclusion is that the system quality improves if we have some prior knowledge available.

\section{REERENCES}

[1] Martz, H.F. and Wallher, R.A. (1982): "Bayesian Reliability Analysis", John Willy, New York.

[2] Bhattacharya, S.K. (1967): "Bayesian Approach to lifetesting and reliability estimation”, JASA, 26, PP. 48-62

[3] G.G. Brush, (1986): “A comparison of classical and Bayes producer's risks", Technometrics 28 (1), 69-72.
[4] G.G. Brush, H. Cautin and B.R. Lewin (1981): “'Outgoing quality distributions for MIL-STD-105 D sampling plans", Journal of Quality Technology, 13, 254-263.

[5] K. K. Sharma and R.K. Bhutani, (1992 c): “A comparison of classical and Bayes risks when the quality varies randomly", Microelectron Reliability, Vol. 32, No. 4, pp. 493-495.

[6] Sharma, K.K. and Bhutani, R. K. (1993): "Bayesian analysis of system availability", Microelectron Reliab., $33,6,809-811$

[7] Sharma, K.K. and Bhutani, R.K. (1992): "A comparison of classical and Bayes risk", Ind. Appl. Statistics, 1, 27-36.

[8] Sharma, K.K. and Bhutani, R.K. (1994): "Bayesian analysis series system”, Microelectron Reliab., 34, 4, 761-763.

[9] Sharma, K.K. and Krishna, Hare (1994): "Bayesian reliability analysis of k-out-of-m system and the estimation of sample size and censoring time", RESS, 44, 11-15.

[10]Box,G.E.P.and Tiao(1973),"Bayesian inference in statistical analysis.

[11] Bazovsky, I.(1961), "Reliability theory and practice", Practice Hall, New Jersey. 